\title{
A Virtual Reality Simulator Based on Haptic Hard Constraints
}

\author{
Julien Castet and Jean-Loup Florens \\ Laboratoire ICA, ACROE \\ 46 avenue Felix Viallet, Grenoble, France \\ \{julien. castet, jean-loup.florens\} @imag.fr
}

\begin{abstract}
This paper presents a real-time physically based platform for multi-sensory interactive simulation. It is centred on high quality dynamic requirements driven by the concept of instrumental interaction and is able to render a wide variety of physical phenomena : from very rigid interactions to large and complex phenomena such pastes or plastic soils. The platform consists in a precisely synchronized multiprocessor architecture extended with a DSP board used for the simulation of very reactive models. It aims for obtain simulation rate necessary for rigid object $(1 \mathrm{KHz}$ and more) in the context of 6 Degree of Freedom.
\end{abstract}

Keywords: Virtual-Reality, Instrumental, Real Time, Synchronisation, Multi Frequency, Multi Processor, Mass Interaction.

\section{Introduction: Instrumental Virtual Reality}

The introduction of haptic devices in VR platforms oriented towards manipulation has motivated lots of active researches the last 20 years. Today, everyone is in agreement with the fact that human interaction with an object necessarily involves mechanical coupling and exchange of mechanical energy. Claude Cadoz first coined this particular property of human gesture under the term 'ergotic' [1] and HCI science has already shown that performance of human-computer interaction could be greatly improved by the use of haptic interfaces 2 .

\subsection{The Haptic Component in Classical Virtual Reality}

In the field of Computer Graphics, like the geometrical modelling and light modelling, physically based modeling stage is also added in the simulation like an independent part to enhance the VR platform with haptic devices.

Within this approach several positions can be found, corresponding to different modelling paradigms of the instrumental chain. Currently, it is commonly admitted that the flow of data between the haptic device and the simulation process must be exchanged at a sampling rate of $1 \mathrm{kHz}$. Therefore this simulation process includes a local mechanical simulation of the virtual object directly

M. Ferre (Ed.): EuroHaptics 2008, LNCS 5024, pp. 918923 2008.

(C) Springer-Verlag Berlin Heidelberg 2008 
related to the haptic device. Due to the focus on graphic synthesis constrains, only this part of the simulation process is running at such frequency, and the rest of the simulation process is running at lower frequency asynchronously of the mechanical model. The mechanical simulation is classically the subject of different interpolations. Below some examples of VR platform architecture can be categorized depending on their computation load repartitions.

SPORE (Simulation of Physically based Objects for Real-time Environments) is an example of "centralized approach" for surgical simulation 3. The simulator is composed of three units: a mechanical unit, a visual unit and a collision unit that run on one general-purpose $\mathrm{PC}$ with a Phantom haptic device. A minimal kernel is dedicated to common processes: ODE and collision detection. A part of the kernel allows to use a collection of complex physically based models.

SPRING is a surgical simulation system dedicated mainly for Collaborative task 4 based on the "Client-Server" configuration. The simulation process runs on a single computer, haptic and audio devices are connected to the simulator through Ethernet network and visualization is duplicated on displays.

Finally there is the "distributed approach" platform where simulation runs on PC cluster. SIMNET [5] was one of the first platforms using the distributed approach; it was a multi-users platform for shooting training. We can also cite FlowVR [6], a development library for network communication, providing tools for the development of interactive applications on clusters of general-purpose PCs.

This implementation strategy is interesting in a large panel of situations because it leads to an efficient use of computation resources, and to suitable, yet not satisfying, solutions for the user, from the point of view of gesture interaction.

\subsection{System Constraints for Instrumental VR}

In instrumental VR, the relation between the gesture interface and the simulated object is not based on phenomenological information, but rather on a bidirectional exchange of data flows that are synchronized with the simulation process. Dynamics of the simulated physical phenomena should be correctly represented into computer simulation, both in terms of simulation frequency and temporal latencies:

- The bandwidth of the simulation should encompass the cut-off frequency of the simulated physical phenomenon: if the simulated model includes acoustical parts, simulation bandwidth should be high enough to generate acoustical frequencies of the sound signal (10 to $50 \mathrm{kHz}$ ).

- The simulation process should be synchronized with input and output of devices involved in the interactive simulation, such as transducers (haptic devices, loudspeakers, etc). Especially, I/O latency of the simulation process should not exceed one simulation period as latency introduces physical distortion [8]. Time 
determinism is the only way to guaranty I/O latency: a step of simulation must be computed within fixed time windows.

\section{Architecture of the Platform Satisfying Presented Constrains of Ergotic Tasks}

\subsection{The Modeling Framework}

The ACROE team has designed since 1984 computer formalism, called the CORDIS ANIMA system [7], based on discrete mass-interaction modelling. A physical object or a set of physical objects are modeled and simulated as a network where the nodes are the smallest modules representing inertia (the MAT elements) and where the links (the LIA elements) represent physical interactions between them. The modules are all implemented with explicit algorithms, allowing for deterministic computation. Thus our multi-sensory simulations are based on one model composed of a large number of simple algorithms allowing a regular computation synonymous of determinism. The input/computation/output sequence can also be easily synchronized on an external clock. Simulation frequency can be adjusted between 1 and $50 \mathrm{kHz}$ according to the bandwidth of the targeted physical phenomenon.

\subsection{Hardware Components}

One of the hardware components is multi-processor (bi or quadri-processors) computer from Concurrent Computer. Processors used are AMD Opteron $2 \mathrm{GHz}$ with cache of $1 \mathrm{Go}$ and 64 bits architecture. The computer is equipped with a Real-Time Clock and Interrupt Module, a PCI Board, which provides a modular synchronization (from external clock or for the synchronization of external modules). Computer uses the Operating System RedHawk, distribution linux specialized for Real-time applications, 9] allow satisfying time determinism requirements.

A DSP PCI board from Innovative Integration, called TORO board, is the second main component of the platform. The embedded DSP is the TMS320C6711 characterized by a computation frequency of $150 \mathrm{MHz}$. This card provides 16 simultaneous analog inputs and outputs up to $250 \mathrm{KHz}$ each, both at 16-bit resolution for high quality haptic. A/D and D/A converters are synchronized on the same clock signal as the simulation process and are used for the exchange of data with the haptic device from ERGOS panoply. Considering that commercial sound boards present non negligible latencies, we have also chosen to take benefit of the 16 bits precision D/A converters for sound outputs, allowing less than 5 us of latencie for a simulation frequency of $44,1 \mathrm{kHz}$.

These hardware components could be used together or independently providing a range of configurations for various performances. The presented platform consists in the complete configuration of the simulator allowing simulations of complex scenes with high reactivity of haptic modality. 


\section{Main Results of the Multiprocessor and Multi-frequencies Configuration}

This new force feedback Virtual Reality Platform has been designed to be able to render a wide variety of physical phenomena : from very rigid interactions to large and complex 3D objects such pastes or plastic soils. The physical manipulation of such objects requires to have at disposal $6 \mathrm{DoF}$ force feedback devices. When using a 6 DoF device, the cinematic transformation becomes more complex, increasing the difficulty to have high rigidity. In order to obtain a very rigid behavior of the force feedback device itself, our platform allow to control it at a frequency higher than the usual frequency of $1 \mathrm{KHz}$.

The realization of this configuration has been organised according two axes. In the first time, work has been focused on the model distribution between the processing units sharing memory(Fig. (1), and next on the multi-frequencies implementation.

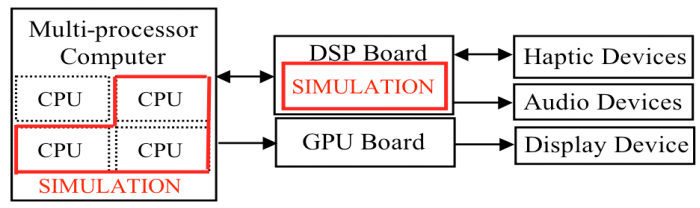

Fig. 1. Configuration with multi-processor computer and DSP Board

\subsection{First Stages of Platform Realization}

The objective of first stage was to obtain a synchronized and mono frequency simulation along the chain of computation. In order to avoid modelling problem and focus on the architecture problem, a simple bypass has been realized on the complete chain of computation at $20 \mathrm{KHz}$ (Fig. 2.a). The input and the output signals are strictely synchronized and phase shifted of one sampling period. The synchronization of computing units is based on active waiting. This allows to ensure an I/O latency of the simulation process which doesn't exceed one simulation period, and a communication between processing units realized in a fixed time window.

The second stage concerns the multi-frequencies computing. In order to obtain a platform with high reactivity and a high computation power, one computing unit must run at high frequency, in this case the DSP, and others CPU at low frequency. This realization is based on two points, or dates, of synchronization per simulation cycle. Naturally, the signal coming from CPU to DSP is also under sampled (impulse under-sampling: see Fig. 2,b).

To validate rigidity of simulation chain, the first realized physical model is the coupling of two oscillators, one on DSP at $5 \mathrm{KHz}$, one on CPU at $1 \mathrm{KHz}$ (Fig. (3)). 

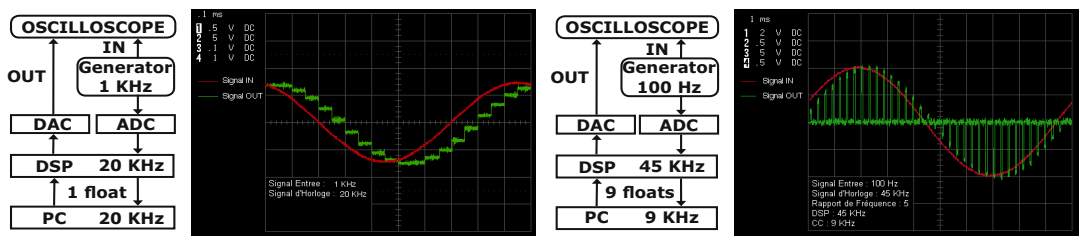

Fig. 2. Bypass bi-processor: a. Monofrequency / b. Bifrequency
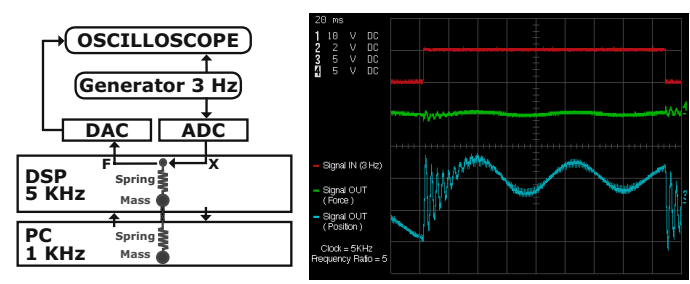

Fig. 3. Elementary model: Impulse Response of 2 oscillators coupled, one per processor

\subsection{The First Simulation of a Complex Scene with High Reactivity of Haptic Modality: La Crepe}

These first implementations aim at obtaining, in the context of a 6 DoF, force feedback manipulation corresponding to the simulation rate that it is necessary for rigid objects ( $3 \mathrm{KHz}$ and more). In order to illustrate the success of the realization, the first simulated scene is what it has been called crêpe , composed of a frying pan and a pancake. This object needs really a 6 DoF force feedback manipulation : to toss the pancake with a complex movement composed by coordinated translations and rotations of the pan. The frying pan must have a very rigid behavior between the manipulated point to the plate which receives the pancake. This scene is a good benchmark for the simulation capability as well as for the haptic requirements in the sense that it is composed of (1) a soft object that has to exhibit complex behaviors, (2) a very rigid object and (3) 6D manipulation of the rigid object aiming at deforming and tossing the soft object. The entire scene (pan, pancake and 6 DoFs cinematic transform) is modelling with only one mass-interaction model providing our simulation performances.

Quantitatively, the batter consists in 190 particles simulated on the bi-processor, that constitute a completely connected network(17955 interactions) where the
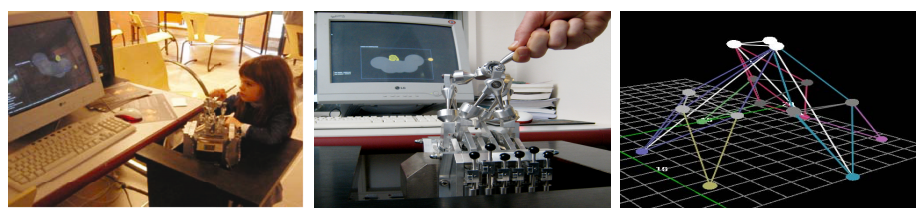

Fig. 4. Screenshots and pictures of the first simulated scene: La crêpe 
interactions are defined by a viscosity and an elasticity with independent thresholds. The pan is simulated with 8 masses on the bi-processor too. The 6 DoF cinematic transformer (Fig. (4) is calculated on the DSP at $9 \mathrm{KHz}$ by an internal clock of DSP Board, and the bi-processor is slaved at 3KHz. Thanks the high performances of the architecture in terms of synchronization and computational power, users have really the possibility to co-articulate complex manipulations such translate-turn-translate in a very efficient mode to toss a deformable object. In our knowledge, such very enactive task has never been obtained.

\section{Conclusion}

Unlike other Virtual Reality platforms, this new real-time physically based hardware platform allows a complete synchronous multi-sensory interactive simulation. Its hardware modular architecture provides a range of configuration adapted to different issues (individual use, laboratory use..). According to performances obtained with the model presented above, the connection between the multiprocessor computer configuration and the DSP board configuration is a really promising solution for a future virtual reality platform allowing the simulation of complex scene with high quality of haptic interaction. Actually, different works are in development : 3D models (pan, mortar and pestle..), cinematic transform library (2D guided plan, guided circle...).

\section{References}

1. Cadoz, C.: Le geste, canal de communication homme/machine. la communication instrumentale. Technique et Science de IInformation 13(1), 31-61 (1994)

2. Rosenberg, L.B., Brave, S.: Using force feedback to enhance human performance in graphical user interfaces. In: Proceedings of CHI 1996, Canada (1996)

3. Meseure, P., et al.: A Physically-Based Virtual Environment dedicated to Surgical Simulation, IS4TM (June 2003)

4. Montgomery, K., et al.: Spring: A general Framework for Collaborative, Real-time Surgical Simulation. In: Medicine Meets Virtual Reality, Amsterdam (2002)

5. Calvin, J., et al.: The simnet virtual world architecture. In: IEEE Virtual Reality Annual International Symposium, IEEE Virtual Reality Annual International Symposium, pp. 450-455 (1993)

6. Dequidt, J., et al.: Collaborative interactive physical simulation. In: GRAPHITE 2005, Dunedin, New Zealand (November-December 2005)

7. Florens, J.-L., Cadoz, C.: The physical Model, Modelisation and Simulation Systems of the Instrumental Universe. In: Representation of Musical Signals, pp. 227-268. The MIT Press, Cambridge (1991)

8. Florens, J.-L., Urma, D.: Dynamical issues at the low level of human / virtual object interaction. In: DBL, Arlington, VA, USA, p. 47 (2006)

9. Castagne, N., Florens, J.-L., Luciani, A.: Computer Platforms for Hard-Real Time and High Quality Ergotic Multisensory Systems - requirements, theoretical overview, benches. In: Proceedings of ENACTIVE 2005, Genoa, Italy (2005) 